\section{CLICKABLE, BIOCOMPATIBLE AND FLUORESCENT HYBRID NANOPARTICLES FOR INTRACELLULAR DELIVERY AND OPTICAL IMAGING}

\author{
Markus Müllner*, Anja Schallon ${ }^{\dagger}$, Andreas Walther*, Ruth Freitag ${ }^{\dagger}$, \\ and Axel H.E. Müller* \\ * Macromolecular Chemistry II, 'ंBioprocess Tech. \\ University of Bayreuth \\ 95440 Bayreuth, Germany
}

\section{Introduction}

The development of biocompatible nanoparticles for molecular imaging and targeted therapy is an area of considerable current interest. ${ }^{1-5}$ Fluorescent nanoparticles have immense potential in a number of biotechnological applications such as biological imaging or sensor technology. ${ }^{6-8}$

In this study, we aim to combine some of the most attractive approaches for biomedical carriers, i.e. self-assembly of well-defined polymers into nanoscopic colloids, their stabilization using a silica-like network in the core with entrapped dye molecules and a protein-repellent periphery, which can be functionalized via click chemistry to bind cell specific ligands. After studying the formation of the micelles and their crosslinking, the nanoscopic carriers are tested with respect to their compatibility and cellular internalization on lung cancer cells. The accessibility of the click function will be demonstrated by the conjugation to another fluorescent dye.

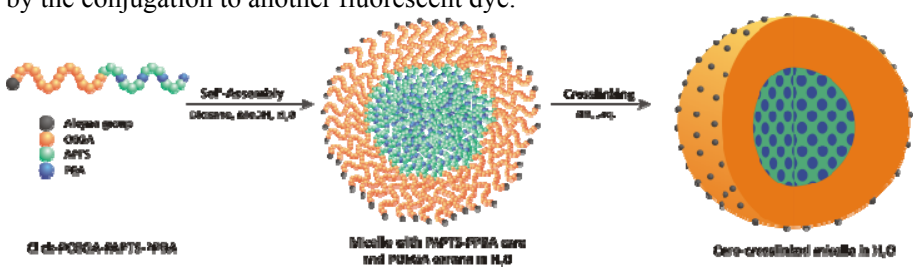

Figure 1. Schematic illustration for the preparation of clickable hybrid nanoparticles via self-assembly and crosslinking in $\mathrm{H}_{2} \mathrm{O}$.

\section{Experimental}

Materials. Benzene (anhydrous), 1-pyrenebutanol (99\%), 2-propyn-1ol (propargyl alcohol, 99\%), triethylamine (TEA, >99\%), 4-dimethylamino pyridine (DMAP, 99\%), N,N'-dicyclohexylcarbodiimide (DCC, 99\%), potassium ferricynide $\left(\mathrm{K}_{3} \mathrm{Fe}(\mathrm{CN})_{4}, 99 \%\right)$, rhodamine B (dye content $\sim 95 \%$ ), 3-chloro-1-propanol $(98 \%)$, and sodium azide $(>99 \%)$ were purchased from Sigma-Aldrich and used without further purification. Acryloyl chloride (97\%), benzylmagnesium bromide solution (1M in THF), and carbon disulfide (99\%) were purchased from Fluka and used as received. Dichloromethane (DCM), methanol, benzene, and dioxane were used in p.a. grade. N,N-Azobisisobutyronitrile (AIBN) was ordered from Aldrich and recrystallized from ethanol. Oligo(ethylene glycol) acrylate (OEGA) with number-average weight $\mathrm{M}_{\mathrm{n}}=454 \mathrm{~g} / \mathrm{mol}$ was purchased from Aldrich and freed from inhibitor by passing through a basic alumina column. (3-Acryloxypropyl) trimethoxysilane (APTS, 95\%) was purchased from ABCR and freed from inhibitor by passing through a silica column. A549 epithelial cells were purchased from LGC Standards ATCC (Wesel, Germany) and were cultured in DMEM (PAA, high glucose) supplemented with $10 \%$ FCS, streptomycin $(100 \mu \mathrm{g} / \mathrm{ml})$, penicillin

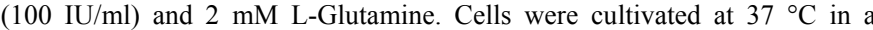
humidified $5 \% \mathrm{CO}_{2}$ atmosphere.

Instrumentation. ${ }^{1} \mathrm{H}$ NMR was used to determine molecular weights. Gel permeation chromatography (GPC) was used to determine molecular weight distributions, $M_{w} / M_{n}$, of polymer samples with respect to tBA standards. Dynamic light scattering (DLS) was used to determine the hydrodynamic radii of the assembled aggregates. Cryogenic transmission electron microscopy (Cryo-TEM) was used to confirm the dimensions of the assembled aggregates. The system configurations have been described previously. ${ }^{9}$ Cells were observed with an epifluorescence microscope (Olympus BX51TF, Hamburg, Germany).

Polymerizations. Synthesis of w-alkyne-poly[oligo(ethylene glycol) acrylate] (PEOGA-CTA). A mixture of propargyl (4-cyanopentanoic acid)-4dithiobenzoate) $(0.25 \mathrm{~g}, 0.8 \mathrm{mmol})$, oligo(ethylene glycol) acrylate (OEGA, $107 \mathrm{~g}, 240 \mathrm{mmol})$ and anhydrous benzene $(100 \mathrm{~mL})$ in a screw-cap flask, sealed with a rubber septum, was deoxygenated by bubbling with nitrogen. In a second flask, a stock solution of AIBN (30 mg, $0.18 \mathrm{mmol}$ ) in anhydrous benzene $(10 \mathrm{~mL})$ was deoxygenated similarly. Afterwards, $8.8 \mathrm{~mL}$ of this stock solution were transferred to the reaction mixture with a syringe and the polymerization flask was placed into an oil bath at $65{ }^{\circ} \mathrm{C}$. Samples were withdrawn to monitor conversion. The polymer was purified via dialysis in dioxane. Freeze drying yielded the final pure marco-CTA.

Synthesis of $\omega$-alkyne-poly[oligo(ethylene glycol) acrylate]-b-poly[(3acryloxypropyl)-trimethoxysilane]-co-poly(1-pyrenebutyl acrylate). Alkynefunctionalized poly[oligo(ethylene glycol) acrylate]-CTA precursor $(4.5 \mathrm{~g}$, $0.092 \mathrm{mmol}, \mathrm{M}_{\mathrm{n}}=48.000 \mathrm{~g} / \mathrm{mol}$ ) was dissolved in anhydrous benzene. To the mixture, (3-acryloxypropyl) trimethoxysilane $(8.7 \mathrm{~g}, 37 \mathrm{mmol})$ and 1pyrenebutyl acrylate $(3.02 \mathrm{~g}, 9.2 \mathrm{mmol})$ were added. The solution was degassed by bubbling with nitrogen. After initiation with AIBN the polymerization flask was placed into an oil bath at $70^{\circ} \mathrm{C}$.

The synthesis of the chain transfer agent was conducted similarly as reported earlier by Goldmann et al. ${ }^{10}$ The synthesis of azido-Rhodamine B was accomplished via esterification of Rhodamine B with an azide functioncarrying alcohol.

\section{Results and Discussion}

Synthesis and Molecular Characterization of Block Copolymers. Oligo(ethylene glycol) acrylate (OEGA) was chosen for polymerization of the first block because of its excellent protein repellence. Its good solubility in most common organic solvents and water guarantees good stabilization of the later aggregates in both organic solvents and water and allows for further reactions in a variety of solvents. (3-Acryloxypropyl)trimethoxysilane (APTS) was chosen for polymerization of the second block due to its ability to undergo crosslinking into a stable, inorganic silsesquioxane network under basic conditions. Additionally, 1-pyrenebutyl acrylate (PBA) was added as a comonomer (molar ratio APTS:PBA $=4: 1$ ) to introduce fluorescent tags into the hydrophobic block. It can furthermore be considered as a hydrophobic drug mimic.

Colloidal Aggregates (Self-Assembly and Crosslinking). The selfassembly process was conducted via step-wise solvent exchange from dioxane to methanol and then to water $(\mathrm{pH}=7, \mathrm{c}=0.1 \mathrm{~g} / \mathrm{L})$. DLS measurements of all aggregates $\left(\mathrm{c}=0.1 \mathrm{~g} / \mathrm{L}, 90^{\circ}\right)$ showed a continuous increase of the hydrodynamic radius $R_{h}$ with increasing block lengths of the second hydrophobic. The hydrodynamic radii range from 14-25 nm. This observation is supported by the results of cryo-TEM measurements (Figure 2). The images show spherical micelles with a moderate size-distribution. Consequently, the size of the micelles can be tuned by changing the length of the hydrophobic block.
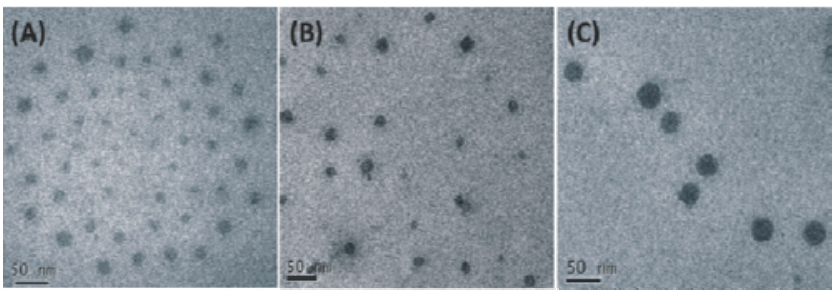

Figure 1. Cryo-TEM images of crosslinked aggregates in water $(\mathrm{c}=0.1 \mathrm{~g} / \mathrm{L})$. The aggregates were formed out of POEGA107-(PAPTS62-PPBA17) (A), POEGA107(PAPTS80-PPBA25) (B) and POEGA107-(PAPTS162-PPBA45) (C)

The micelles were crosslinked into a stable silica-like core with a silsesquioxane network via a mild treatment with ammonia (3\% v/v, 2 days, RT). The crosslinked PAPTS domains lock the shape and additionally embed the organic dyes into the inorganic and rigid silsesquioxane network. Indeed after the reaction, the shape of the resulting aggregates is preserved as revealed by DLS. The core-stabilized micelles do even not disassemble in good organic solvents for both blocks, thus demonstrating the tight crosslinking within the core.

Optical Properties and Click Functionalization. Fluorescence and $\mathrm{UV}-\mathrm{V}$ is measurements give a proof of the incorporation of the dye within the polymer and later aggregates. The measurements demonstrate the excellent fluorescent properties of pyrene as a biomarker. Furthermore, pyrene can be taken as a general example for any hydrophobic cargo conjugated into the micelle core. 
The accessibility of the click function at the periphery of the nanoparticles was assessed via a conjugation of a second dye. The successful clicking of azido-functionalized Rhodamine B onto the nanoparticles highlights the possibility of further modifying the nanoparticles in a facile and orthogonal fashion. Clicking Rhodamine B onto the polymer aggregates represents a simple model reaction for our easy and versatile approach of modifying nanoparticles. UV-Vis characterization of the polymer aggregates before and after the click reaction demonstrates the successful attachment of Rhodamine B via the azide-alkyne Huisgen cycloaddition (Figure 3 A, C) Whereas the pristine nanoparticle solutions did not show any absorption in the range of 520-600 nm, a peak induced by Rhodamine B was observed around those wavelengths after the click reaction. Emission fluorescence spectroscopy analysis of polymer aggregate solutions before and after clicking gave concurrent evidence.
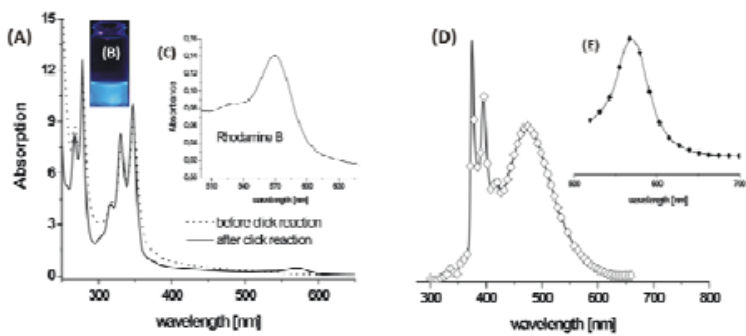

Figure 2. UV-Vis spectra (left) of crosslinked nanoparticles of POEGA107-(PAPTS68 PPBA20) in aqueous solution $(\mathrm{c}=0.1 \mathrm{~g} / \mathrm{L})$ : (A) UV-Vis spectra before (dotted line) and after (solid line) clicking with azido-functionalized Rhodamine B. (B) Non-clicked nanoparticle solution exposed to UV light. (C) Magnification of Rhodamine B absorption from (A). Emission Fluorescence spectra (right) of crosslinked nanoparticles of POEGA107-(PAPTS68-PPBA20) in aqueous solution ( $c=0.1 \mathrm{~g} / \mathrm{L}$ ): (D) before modification with Rhodamine B, excitation wavelength: $275 \mathrm{~nm}$; (E) after clicking with azido-Rhodamine B, excitation wavelength: $510 \mathrm{~nm}$.

Biocompatibility and Cell Distribution. To assess the biocompatibility and the distribution of the nanoparticles within cells, studies were carried out with lung cancer cells. For this purpose, we performed mitochondria toxicity test (MTT) assays and light microscopy imaging of cells treated with different solutions. Both crosslinked and non-crosslinked polymer aggregates based on POEGA107-(PAPTS62-PPBA17), POEGA107-(PAPTS68-PPBA20), and POEGA107-(PAPTS80-PPBA25) were used. All three hybrid particles showed no significant or even lethal effect on vitality (more than $90 \%$ of the cells remained unaffected). Even upon adding about $30 \mathrm{vol} \%$ of nanoparticle solution to the cell media, the vitality remained unchanged. This high compatibility can be ascribed to our choice of POEGA as outer block.

After these promising toxicity results, we focused on the cellular distribution of the nanoparticles after adding them to cells. The incorporated pyrene allows an easy localization of the nanoparticles within the cells. Epifluorescence microscopy images show a strong presence of nanoparticles near the cell nucleus, whose DNA was stained with the green dye SYTOX®GREEN (Figure 4A and C). Since the nanoparticles are labelled with Rhodamine B as well, red fluorescence (Figure $4 \mathrm{D}$ ) can be detected at the same positions where the blue fluorescence of pyrene (Figure $4 \mathrm{~B}$ ) is emitted, nicely confirming the successful click modification of the particles. Note that the nanoparticles contribute some intensity to the green filter channel due to their brighter fluorescence and high concentration. The close proximity of the nanoparticles to the nucleus could be further seen in transmission mode (Figure $4 \mathrm{E}$, arrows). The polymer shows interaction with the cell, but not with the DNA (Figure 4 A-D). Accumulations of polymers were detectable around the nucleus, whereas the nucleus showed no fluorescence (Figure $4 \mathrm{~B}$, arrows). To reach the green stained nucleus (Figure $4 \mathrm{~F}$ ), the particles first have to permeate through the cell membrane into the cell. Accordingly it is possible with our nanoparticles to deliver matters into cells and very close to the nucleus itself. This could be an advantage for biotechnology and drug delivery since interaction with the DNA can lead to mutations. With this polymer drugs can be delivered into the cell without being toxic, even at high concentrations. Additionally it can be chosen whether matters should be delivered protected in the micelle core or rather on the outer surface. By that fact, the system incorporates a high flexibility and possibility for adjustments depending on the specific use of the nanoparticles or nanocarriers.
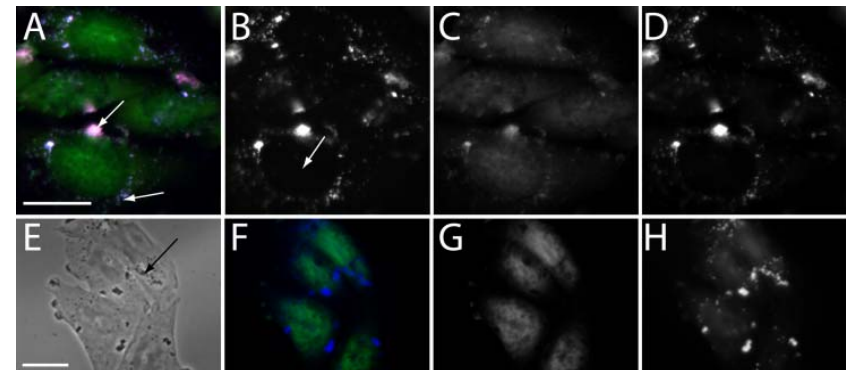

G

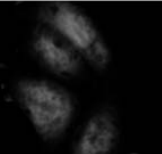

Figure 3. Microscope images of A549 cells exposed to Rhodamine B functionalized nanoparticles based on POEGA107-(PAPTS62-PPBA17). (A) merged optical microscope image of the fluorescence images of B, C and D. (B) and (D) exclusively show the red (rhodamine B) and blue (pyrene) fluorescent parts, respectively. (B) and (D) resemble the distribution of the nanoparticles. (C) shows the green stained (SYTOX ${ }^{\circledR}$ GREEN) DNA parts, primarily the nucleus. Rhodamine labeled POEGA107-(PAPTS62-PPBA17) allowed to study the adsorption of nanoparticles in the cells. (E) Transmission microscope image of cells: the nanoparticles (dark dots) arrange near the nucleus. $(\mathrm{G})$ exclusively shows the green light emitting parts (nucleus), whereas $(\mathrm{H})$ exclusively shows the blue light emitting channel (nanoparticles, POEGA107-(PAPTS62-PPBA17)). (F) shows a merged microscope image of $(\mathrm{G})$ and $(\mathrm{H})$. The scale bars resemble $20 \mu \mathrm{m}$

\section{Conclusions}

The $\mathrm{Cu}(\mathrm{I})$-catalyzed azide-alkyne cycloaddition (CuAAC) has recently proven to be a powerful synthetic tool in various fields of chemistry, including protein-polymer conjugation. In this work we present new, bright fluorescent, and biocompatible carriers derived from well-defined amphiphilic block copolymers possessing a crosslinkable part and pyrene as an incorporated dye. RAFT polymerization allowed an excellent control over the block length of the hydrophobic block, plus the amount of pyrene incorporated. By that, size tuning of the size of later polymeric micelles became just an issue of polymerization time. The copolymerization of a dye-functionalized acrylate and the utilization of a functionalized chain transfer agent enabled the preparation of fluorescent and easily modifiable hybrid nanoparticles of different dimensions. They show excellent solubility in aqueous as well as in organic media. Our nanoparticles, highlight an easy approach of obtaining size-tunable, fluorescent, stable, and surface-modifiable hybrid particles of no significant toxicity. According to their properties, they could emerge as a new class of versatile delivery vehicles or for biosensing. The possibility and versatility of click reactions on the nanoparticles gives great opportunity for surface functionalization and tailored properties for sensing and targeted, nontoxic delivery into cells. Clicking bioconjugates onto the hybrid materials can be an interesting way of labeling bioactive conjugates.

Acknowledgements. The authors of this paper thank the European Science Foundation for support within the SONS 2 program (project BioSONS).

\section{References}

(1) Alivisatos, P. Nat Biotech 2004, 22, 47-52.

(2) Christof, M. N. Angewandte Chemie International Edition 2001, 40, 4128-4158

(3) Michalet X.; Pinaud F.F.; Bentolila L.A.; Tsay J.M.; Doose S.; Li J.J Sundaresan G.; Wu A.M.; Gambhir S.S.; Weiss S. Science 2005, 307, 538-544.

(4) Smith A. E.; Duan H.; Mohs A. M.; Nie S. Advanced Drug Delivery Reviews 2008, 60, 1226-1240.

(5) Nathaniel L. Rosi, C. A. M. ChemInform 2005, 36.

(6) Li, G.; Shi, L.; An, Y.; Zhang, W.; Ma, R. Polymer 2006, 47, 45814587.

(7) Jaiswal, J. K.; Mattoussi, H.; Mauro, J. M.; Simon, S. M. Nature Biotechnol. 2003, 47.

(8) Terai, T.; Nagano, T. Current Opinion in Chemical Biology 2008, 12 , 515-521.

(9) Walther, A.; Goldmann, A. S.; Yelamanchili, R. S.; Drechsler, M.; Schmalz, H.; Eisenberg, A.; Müller, A. H. E. Macromolecules 2008, 41, 3254-3260

(10) Goldmann A.; Quémener D.; Millard P.; Davis T.P.; Stenzel M.H.; Barner-Kowollik C.; Müller A. H. E. Polymer 2008, 49, 2274. 